\title{
Scribing of Thin-Film Solar Cells with Picosecond and Femtosecond Lasers
}

\author{
Paulius Gecys ${ }^{* 1}$, Gediminas Raciukaitis ${ }^{* 1}$, Anja Wehrmann ${ }^{* 2}$, Klaus Zimmer ${ }^{* 2}$, Alexander Braun ${ }^{* 3}$ and Steffen Ragnow ${ }^{* 3}$ \\ ${ }^{* 1}$ Center for Physical Sciences and Technology, Savanoriu Ave. 231, Vilnius, LT-02300, Lithuania \\ E-mail:p.gecys@ar.fi.lt \\ ${ }^{* 2}$ Leibniz-Institute of Surface Modification, Permoserstr. 15, 04318 Leipzig, Germany \\ ${ }^{* 3}$ Solarion AG, Ostende 5, 04288 Leipzig, Germany
}

Continuous growth of the thin-film solar technology market stimulates the development of versatile technologies for large-scale patterning of thin-film materials on rigid and flexible substrates, and laser technologies are a promising method to accomplish the scribing processes.

In this study we compare picosecond and femtosecond pulse laser scribing for thin-film solar cells. For this we selected a Nd:YVO 4 mode locked picosecond laser with the pulse duration of $10 \mathrm{ps}$ and a Ti: sapphire laser with the pulse duration of $300 \mathrm{fs}$. We concentrated on so-called P3 step of laser scribing to expose the molybdenum back-contact. The visual quality of the scribes was controlled with optical and scanning electron microscopes. The conversion efficiency tests, LIT (lock-in thermography) and LBIC (laser-beam induced current) measurements were performed on the laser-scribed complete working solar cells of prefabrication stage.

The damage-free exposure of molybdenum layer was possible in complex thin-film structure with both pulse durations. LIT and LBIC measurements did not show evidence of internal shunt formation near the scribed zone using lasers with both pulse durations. The efficiency tests confirmed an insignificantly higher solar cell performance after femtosecond laser scribing, although at both pulse durations marginal reduction in solar cell efficiency was observed.

DOI:10.2961/jlmn.2012.01.0006

Keywords: thin-film solar cells, CIGS, laser scribing, picoseconds, femtosecond,

\section{Introduction}

Continued demand for renewable energy sources stimulates development of various solar cell technologies on flexible and rigid substrates. The thin-film PV technologies based on $\mathrm{CuIn}_{\mathrm{x}} \mathrm{Ga}_{(1-\mathrm{x})} \mathrm{Se}_{2}$ (CIGS) become more attractive due to their potential in lowering the production cost and optimization of photoelectrical performance. Other properties such as flexibility, good power-weight ratio, resistance to radiation make CIGS solar cells ideal for space use, automotive industry and complex structure building integrated applications.

CIGS has been established as the most efficient thinfilm technology in converting sunlight into electricity with the theoretical limit as high as $27 \%$ [1] and a record value of $20.3 \%$ achieved in the laboratory conditions [2]. The manufacturing costs and cell efficiency are critical factors for the wider applicability in terms of economical point of view.

Efficiency of the thin-film solar cells with a large active area might be maintained if small segments are interconnected in series in order to reduce photocurrent in thin- films and resistance losses. The structuring is accomplished by three critical scribing processes called P1, $\mathrm{P} 2$, and P3. The P1 scribe must penetrate completely through the full thickness of the contact layer next to a substrate. This cut defines and isolates the individual cells with an effective resistance of tens of megaohms. The P2 scribe through the active layer is important for formation of the series inter-connects between cells. The integrity and continuity of the P3 scribe are as critical as of P1 scribe. Cutting through both the top-contact and semiconductor layers, the P3 scribe isolates adjacent cells. Selective damage free removal of the films in multilayer structures of modern solar cells is crucial for performance of the devices.

A comprehensive study of thin film scribing for photovoltaics including CIGS with different types of lasers has been conducted by Compaan et al. [3]. Long nanosecond pulses used were found to be favorable for damage-free exposure of molybdenum in the $\mathrm{CIGS} / \mathrm{Mo} /$ glass structure but excessive melt formation was observed from the CIGS layer itself. The main limiting factor to nanosecond laser processing of the multilayer $\mathrm{CuInSe}_{2}$ (CIS) structures is deposition of molybdenum on walls of channels scribed in the films, and the phase transition of semiconducting $\mathrm{CuInSe}_{2}$ to a metallic state close to the ablation area due to the thermal effect [3]. Both effects create shunts in the photo-electric device and reduce its conversion efficiency. According to the results of theoretical modeling, processing without damage is possible with ultra-short-pulse lasers [4, 5], although there have been reports on $\mathrm{p}-\mathrm{n}$ junction damage working even in the femtosecond regime [6].

Our goal was to develop the flexible and rapid laser technology for precise structuring of the CIGS solar cells compatible with the roll-to-roll production line. We used lasers with the picosecond and femtosecond pulse durations in scribing the CIGS thin-film solar cells deposited on flexible polymer substrate. Evaluation of laser scribe quality, elemental analysis, investigations of local electrical properties of solar cells near the laser scribing zone together with efficiency and parallel resistance measurements are presented for both pulse durations. 


\section{Experimental}

The \#1 laser scribing setup included the picosecond laser with the typical pulse duration of $10 \mathrm{ps}, 1064 \mathrm{~nm}$ wavelength, $6 \mathrm{~W}$ average power at $200 \mathrm{kHz}$ laser repetition rate. The beam delivery path consisted of an electro-optical shutter for fast beam control, the attenuator made of half wave plate and a polarizer, beam expander and galvanometer scanner with $300 \mathrm{~mm}$ focal length f-theta lens for fast laser beam positioning.

The \#2 laser scribing setup included the femtosecond laser with the typical pulse duration of $300 \mathrm{fs}, 775 \mathrm{~nm}$ wavelength, $1 \mathrm{~W}$ average power ant $1 \mathrm{kHz}$ laser repetition rate. The beam delivery path consisted of a mechanical shutter for beam control and the attenuator made of half wave plate and a polarizer. F-theta objective of the $300 \mathrm{~mm}$ focal length was used for the beam focusing. In this case $\mathrm{XYZ}$ linear motion stages were used for precise sample positioning.

Our development was concentrated on the P3 process, scribing of the films, to expose the molybdenum backcontact by selective removal of both the top-contact and CIGS layers. Complete thin-film CIGS solar cell structures were used in ablation and scribing experiments. The frontcontact consisted of $\mathrm{Al}: \mathrm{ZnO}$ deposited on $2 \mu \mathrm{m}$ thickness $\mathrm{CuIn}_{\mathrm{x}} \mathrm{Ga}_{(1-\mathrm{x})} \mathrm{Se}_{2}$ absorber material with a thin $\mathrm{CdS}$ buffer layer in between. The back-contact was made of $1 \mu \mathrm{m}$ thick Mo layer. The flexible polyimide film of $25 \mu \mathrm{m}$ thickness was used as a substrate.

The quality of processing was controlled with optical and scanning electron microscopes together with an X-ray energy dispersion spectrometer (EDS) for elemental analysis of the scribes. Complete working solar cells of prefabrication stage with active area of $32 \mathrm{~cm}^{2}$ were scribed in the area between the front-contact grids as shown in Fig. 1. The main goal was to evaluate alterations in solar cell photo-electrical properties after laser scribing.

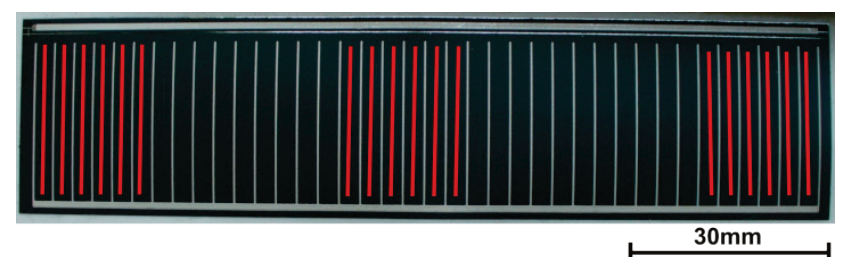

Fig. 1. Red lines indicate laser scribed trenches. The total length of the scribes was $360 \mathrm{~mm}$.

Red lines indicate separate laser scribes of $20 \mathrm{~mm}$ in length and the total scribe length was $360 \mathrm{~mm}$. These solar cells were not final product and did not gain their optimal performance. The photo-electrical efficiency and parallel resistance measurements were performed at irradiance using the standard global spectra AM 1.5 and $1000 \mathrm{~W} / \mathrm{m}^{2}$ intensity. Lock-in thermography (LIT) and Laser Beam Induced Current (LBIC) measurements were performed in the area near the scribes to detect any short-circuiting caused by laser ablation.

\section{Results}

Ablation with a train of laser pulses per spot and scribing lines were performed from the top-contact side to expose the molybdenum back-contact using lasers with both pulse durations. The laser spot overlap along a scanning line was controlled by the translation speed at a constant pulse repetition rate. Various combinations of pulse energy, beam overlap and wavelength were used for selective ablation of the films. Optimal regimes for laser processing of every layer were estimated depending on the pulse duration. The beam overlap or the number of pulses played an important role in the processing selectivity because the ablation threshold was sensitive to accumulation of the irradiation dose.

\subsection{Exposure of the back contact with a burst of laser pulses}

Exposure of the Mo back-contact with several high energy pulses was found not favorable due to pealing of top-contact and extensive melt of CIGS layer while ablation with burst of low energy pulses significantly reduced thermal diffusion into the thin layers and resulted in smooth ablation $[7,8]$. In our research, the ablation with a train of laser pulses per spot was performed from the topcontact side to expose the molybdenum back-contact with both lasers. We used a simple method to determine the back-contact exposure threshold for Gaussian beams [9] by ablating 10 pulses per spot with different laser pulse energies and measuring the crater diameters of the exposed Mo area. Relationships between the exposed area of Mo layer and laser pulse energies are shown in Fig. 2 for both pulse durations.

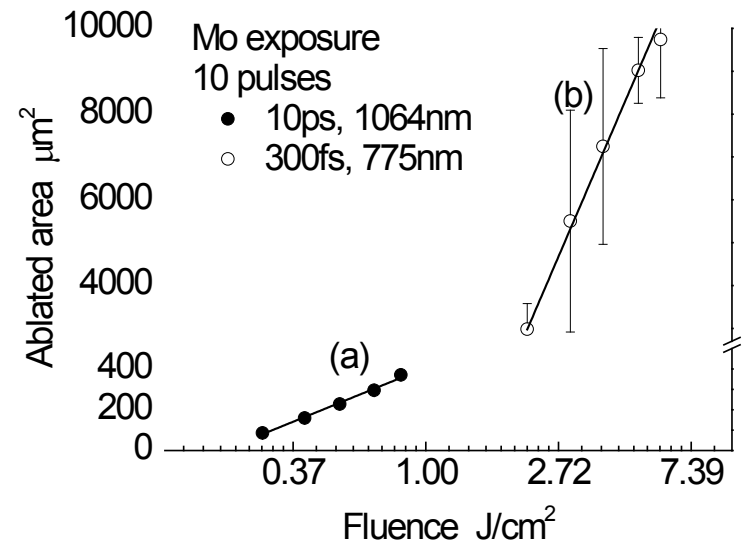

Fig. 2. Relationship between the exposed area of Mo layer and laser fluence: (a) $10 \mathrm{ps}, 1064 \mathrm{~nm}$ wavelength, $200 \mathrm{kHz}$; (b) $300 \mathrm{fs}$, $775 \mathrm{~nm}$ wavelength, $1 \mathrm{kHz}$.

Table 1. The threshold fluencies for exposure of the molybdenum back-contact and beam waist estimated from crater ablation for different laser pulse durations.

\begin{tabular}{ccc}
\hline Pulse duration & $\begin{array}{c}\text { Mo exposure } \\
\text { threshold }\end{array}$ & $\begin{array}{c}\text { Gaussian beam } \\
\text { radius }\end{array}$ \\
\hline $10 \mathrm{ps}$ & $0.22 \mathrm{~J} / \mathrm{cm}^{2}$ & $11.6 \mu \mathrm{m}$ \\
\hline $300 \mathrm{fs}$ & $1.3 \mathrm{~J} / \mathrm{cm}^{2}$ & $62 \mu \mathrm{m}$ \\
\hline
\end{tabular}

For the picosecond pulse duration the lower Mo exposure threshold was found compared to femtosecond pulses and this could be explained by the lower ablation rate of CIGS layer due to lower heat diffusion length at femtosecond pulse irradiation time. This can be the advantage of industrial applications of picosecond lasers where the process speed and efficiency are important. Unfortunately, due to distortions in the beam shape of our femtosecond system no comparative analysis of crater ablation quality could be made for both pulse durations, 
although picosecond pulses showed larger melt area formation near the exposed Mo area.

\subsection{Scribing of CIGS to expose back-contact}

For both picosecond and femtosecond laser systems, the use of high pulse energies with the low pulse overlap caused a poor layer removal selectivity and damage of the Mo back-contact, although more melted CIGS material was observed when scribing with picosecond laser pulses.

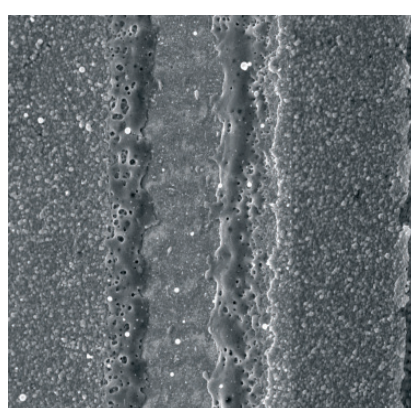

(a)

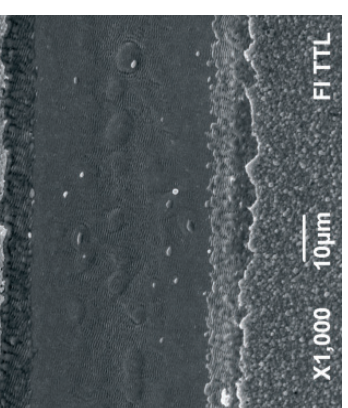

(b)
Fig. 3. Tilted SEM images of the $\mathrm{P} 3$ scribe in $\mathrm{ZnO} / \mathrm{CIGS} / \mathrm{Mo} / \mathrm{PI}$ structure with: (a) $10 \mathrm{ps}, 200 \mathrm{kHz}, 1064 \mathrm{~nm}$ wavelength, scribing speed $1.2 \mathrm{~m} / \mathrm{s}$; (b) $300 \mathrm{fs}, 1 \mathrm{kHz}, 755 \mathrm{~nm}$ wavelength, scribing speed $4 \mathrm{~mm} / \mathrm{s}$.

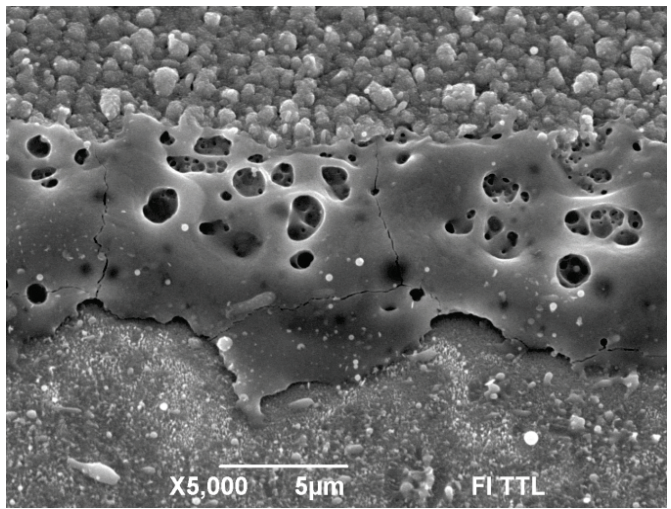

(a) $10 \mathrm{ps}, 1.2 \mathrm{~m} / \mathrm{s}$ scribing speed.

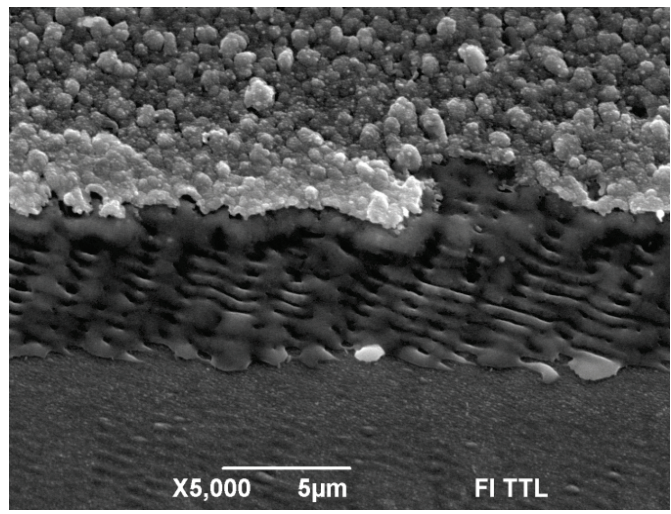

(b) $300 \mathrm{fs}, 4 \mathrm{~mm} / \mathrm{s}$ scribing speed.

Fig. 4. Tilted SEM images of the $\mathrm{P} 3$ scribe edge in $\mathrm{ZnO} / \mathrm{CdS} / \mathrm{CIGS} / \mathrm{Mo} / \mathrm{PI}$ structure with: (a) $10 \mathrm{ps}, 3.5 \mathrm{~W}$, $200 \mathrm{kHz}, 17.5 \mu \mathrm{J} 1064 \mathrm{~nm}$ wavelength, scribing speed $1.2 \mathrm{~m} / \mathrm{s}$; (b) $300 \mathrm{fs}, 35 \mathrm{~mW}, 1 \mathrm{kHz}, 35 \mu \mathrm{J} 755 \mathrm{~nm}$ wavelength, scribing speed $4 \mathrm{~mm} / \mathrm{s}$.

Lowering of the pulse energy and applying higher pulse overlap facilitated an increase in selectivity of layer ablation and reduced melt formation in the CIGS layer for both pulse durations. SEM images of P3 scribe edge in

CIGS solar cell material with $10 \mathrm{ps}$ and $300 \mathrm{fs}$ pulse durations are shown in Fig. 3 and Fig. 4. Even at optimal scribing conditions, the larger melt area formation was observed at the picosecond laser scribe. While scribing with 300 fs laser pulses, the melt area was significantly reduced. Cleaner Mo exposure was also observed at femtosecond regime. The Mo area was not covered with debris from the CIGS layer and smooth Mo surface was observed. This could be explained by better layer selectivity, lower etch rate and "colder" ablation of the material with femtosecond laser pulses. Our previous work [7] revealed that even at higher melt formation of the CIGS layer during picosecond laser scribing no $\mathrm{CuSe}_{2}$ secondary phase was detected with Raman spectroscopy. This metallic phase formation is responsible for short-circuit formation during laser scribing processes with nanosecond lasers. Going to even lower thermal ablation with femtosecond pulses, complete damage-free thin-film solar cell scribing could be realized.

\subsection{X-ray energy dispersion (EDS) analysis}

Layer selectivity and depth control are crucial for the high quality complex structure thin-film solar cell scribing. For the nanosecond regime the main limiting factor of solar cell scribing was projection of melted Mo layer onto the sidewalls of the scribed trench [5] and a significant decrease in solar cell performance.

Zn Cu In Se Mo

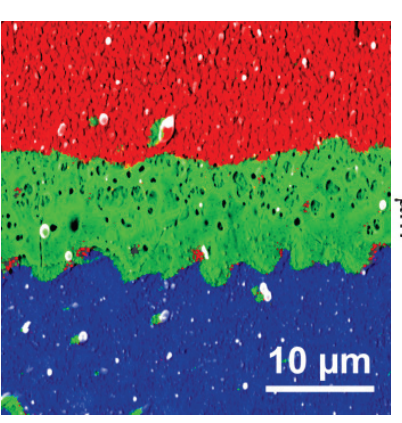

(a) $10 \mathrm{ps}, 1.2 \mathrm{~m} / \mathrm{s}$ scribing speed.

Zn Cu In Se Mo

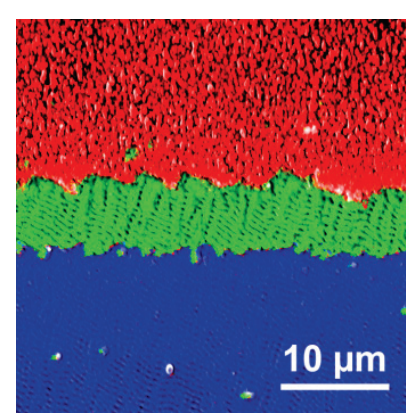

ments, \%

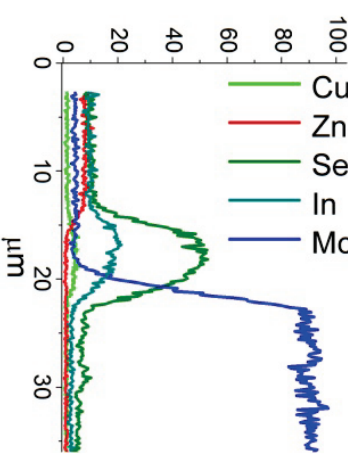

(b) $300 \mathrm{fs}, 4 \mathrm{~mm} / \mathrm{s}$ scribing speed.

Fig. 5. EDS map and cross-section of chemical elements distribution along the yellow line of the P3 scribe edge in $\mathrm{ZnO} / \mathrm{CdS} / \mathrm{CIGS} / \mathrm{Mo} / \mathrm{PI}$ structure: (a) $10 \mathrm{ps}, 1.2 \mathrm{~m} / \mathrm{s}$ scribing speed; (b) $300 \mathrm{fs}, 4 \mathrm{~mm} / \mathrm{s}$ scribing speed. Lateral resolution of the EDS detector was $\sim 1 \mu \mathrm{m}$. 
To investigate the remaining layer structure after picosecond and femtosecond laser scribing, SEM together with X-ray energy dispersion spectrometer measurements were applied. SEM images of investigated area together with a map of chemical elements are presented in Fig. 5. For both pulse durations EDS analysis did not detect projection of molted molybdenum on the edges of the scribed trench and remaining layers still had sharp interfaces. No carbon was detected at the bottom of the scribes in both cases confirming that the Mo back-contact layer was not damaged or cracked. This demonstrates the potential of ultra-short lasers for high quality selective thin layer micromachining.

\subsection{Lock-in thermography measurements}

The main limiting factor to reach higher solar cell efficiencies is the defect accumulation in the absorber material causing internal shunt formation and decrease in photo-current. Laser scribing could lead to defect formation and shunt detection near the laser affected areas is important step to characterize electrical properties of solar cells. Lock-in thermography has proven to be a valuable technique for non-uniformity diagnostics in crystalline and multicrystal-line solar cells $[10,11]$. It utilizes ac infrared imaging of a device, where the temperature is affected by an external ac voltage of the same lock-in frequency. The thermography maps thus represent the current distributions [12]. The lock-in thermography temperature distribution maps are shown in Fig. 6.

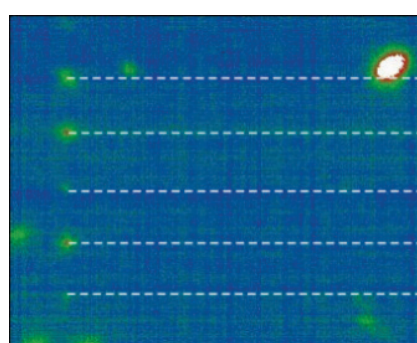

(a)

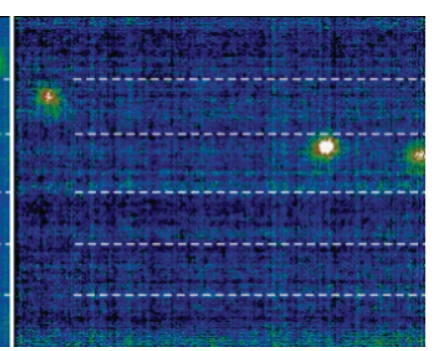

(b)
Fig. 6. Map of temperature distribution in CIGS solar cell area after laser scribing. Blue area represents the colder part of the surface while red color represents warm part. White lines represent laser scribes made using lasers with pulse duration of: (a) $10 \mathrm{ps}$, (b) $300 \mathrm{fs}$.

The film-deposition defects are clearly visible and they cause a short-current leak at the examined surface although these defects were created in the production of the solar cell and are not related to laser scribing. For the picosecond regime some increase in temperature was detected at the end of the laser scribes and this is related to the tune up of the scanning system because the laser beam was closed too late after the motion stopped. In the area where the laser beam was moving at a constant speed no change in the surface temperature was observed. For the femtosecond scribing regime, no temperature change was detected close to the scribing area either. These results conclude that no significant internal shunt formation was detected during laser scribing with both pulse durations. The IR camera was optimized for large area observation and the resolution was too poor to observe area in the range of a scribe width and small defects might not be detected.

\subsection{Laser beam induced current (LBIC) measurements}

Laser beam induced current is nondestructive optical technique for the detection of semiconductor defects [13]. In this technique, a laser beam is applied to the material to induce current to flow through two ohmic contacts placed on the boundary.

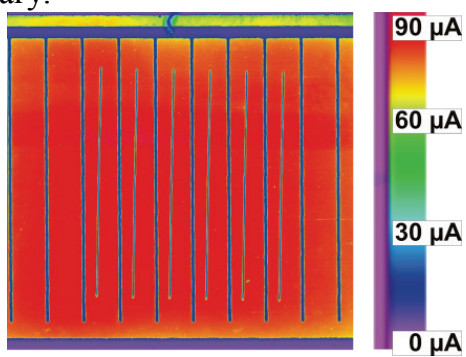

Fig. 7. Photo-current map of the solar cell area after picosecond (10 ps) laser scribing.

The LBIC image consists of measurements of the total local current flowing out through contacts induced by a laser beam applied at laser illuminated location. The diode laser beam was focused into $50 \mu \mathrm{m}$ spot and this defined the resolution of the measurements. The map of LBIC measurement areas close to laser scribe is shown in Fig. 7. Laser scribing was applied between the grids of frontcontact. The LBIC map showed uniform distribution of current in the area close to scribe lines made with picosecond laser. The dead area near the scribe was minimal, although the resolution of our measurements did not allow us to investigate defect formation in micro-scale at the edge of ablated trench.

\subsection{Solar cell performance test after laser scribing}

Complete working solar cells of prefabrication stage with the average efficiency of $10.7 \%$ and the active surface area of $32 \mathrm{~cm}^{2}$ were scribed using optimal scribing parameters with both laser systems. The total length of laser scribes was $360 \mathrm{~mm}$ in all cases. The efficiency tests were performed before and after laser scribing to evaluate influence of laser scribing on the solar cell performance at the standard testing conditions (standard global spectra AM 1.5 and $1000 \mathrm{~W} / \mathrm{m}^{2}$ total irradiance).

Table 2. Photo-electrical efficiency of CIGS solar cells and $\mathrm{R}_{\text {parallel }}$ measurement results before and after laser scribing.

\begin{tabular}{|c|c|c|c|}
\hline & $\begin{array}{l}\text { Absolute eff. } \\
\text { drop, } \%\end{array}$ & & $\begin{array}{c}\text { Absolute } \\
\mathrm{R}_{\text {parallel }} \\
\text { drop, Ohm }\end{array}$ \\
\hline \multicolumn{4}{|l|}{$10 \mathrm{ps}$} \\
\hline$\# 1$ & 0.03 & & 7.09 \\
\hline$\# 2$ & 0.57 & & -0.46 \\
\hline$\# 3$ & 0.44 & & \\
\hline $\begin{array}{c}\text { Average } \\
\text { absolute eff. } \\
\text { drop, } \%\end{array}$ & 0.35 & $\begin{array}{c}\text { Average } \\
\text { absolute } \mathrm{R}_{\text {parallel }} \\
\text { drop, Ohm }\end{array}$ & 3.3 \\
\hline \multicolumn{4}{|l|}{$300 \mathrm{fs}$} \\
\hline$\# 4$ & 0.78 & & -1.36 \\
\hline$\# 5$ & 0.01 & & -0.75 \\
\hline$\# 6$ & -0.08 & & 26.49 \\
\hline $\begin{array}{c}\text { Average } \\
\text { absolute eff. } \\
\text { drop, } \%\end{array}$ & 0.24 & $\begin{array}{c}\text { Average } \\
\text { absolute } \mathrm{R}_{\text {parallel }} \\
\text { drop, Ohm }\end{array}$ & 8.13 \\
\hline
\end{tabular}


Three solar cells were scribed with 10 ps laser pulses and also three cells were processed with the $300 \mathrm{fs}$ pulsed laser. The results of efficiency and parallel-resistance measurements are shown in Table 2. We used a definition of an absolute average drop of the solar cell initial efficiency which is equal to the difference of efficiency before and after laser scribing process including spontaneous efficiency degradation of solar cells in time due to contact with ambient air. The efficiency tests after laser scribing showed an insignificant decrease in solar cell performance and parallel resistance during laser scribing with both pulse durations. Solar cell samples processed with the femtosecond laser showed marginally better performance. This could be related to the less thermal ablation of the CIGS layer with femtosecond pulses, although still defect formation near the laser affected zone occurred.

\section{Conclusions}

For P3 scribing of thin-film CIGS solar cell a picosecond laser showed a better layer removal efficiency although greater thermal impact on the thin layers was observed compared to femtosecond regime. Selective and damage-free laser scribing was possible for the Mo backcontact exposure with both pulse durations. The solar cell efficiency test revealed marginal degradation in efficiency after the laser scribing was applied to the solar cell samples. Even using ultrashort pulsed lasers for scribing GIGS material, the narrow melted edge and laser affected area were formed due to Gaussian distribution of the laser beam and most likely in these zones generation of shunts and defects provoked reduction of electrical properties of investigated solar cells. LIT and LBIC measurements were not able to detect any defect areas mainly due to inadequate resolution of the devises. Deeper analysis of laser scribing effect on photo-electrical performance of solar cells requires higher resolution to determine shunt formation areas. To decrease defect and shunt formation during laser scribing process two approaches are possible: to use a tophat shaped beam and minimize the laser affected area or (and) to use shorter pulse durations than $300 \mathrm{fs}$.

\section{Acknowledgments}

The research was supported by the Research Council of Lithuania under project No AUT-06/2010.

The internship of P. Gecys to the Leibniz Institute for Surface Modification was supported by Research Council of Lithuania under project "Promotion of student academic activity".

\section{References}

[1] T. Cheyney: Photovoltaics International, 1, (2008) 86.

[2] J. Kho: Photovoltaics World, 6, (2010) 24.

[3] A. D. Compaan, I. Matulionis, S. Nakade: Optics and Lasers in Engineering, 34(1), (2000) 15.

[4] J. Hermann M. Benfarah, S. Bruneau., E. Axente, G. Coustillier., T. Itina, J.-F. Guillemoles, P. Alloncle: Journal of Physics D: Applied Physics, 39(3), (2006) 453.

[5] J. Hermann, M. Benfarah, G. Coustillier, S. Bruneau, E. Axente, J.-F. Guillemoles, M. Sentis, P. Alloncle, T. Itina: Applied Surface Science, 252(13), (2006) 4814.
[6] A. Wehrmann, H. Schulte-Huxel, M. Ehrhardt, D. Ruthe, K. Zimmer, A. Braun, S. Ragnow: Proc. SPIE, 7921, (2011) 79210T.

[7] P. Gečys, G. Račiukaitis, E. Miltenis, A. Braun, S Ragnow: Physics Procedia, 12 (2), (2011) 141.

[8] P. Gecys, G. Raciukaitis, M. Erhardt, K. Zimmer, M. Gedvilas, Appl. Phys. 101 (2010) 373.

[9] J. M. Liu: Optics Letters, 7(5), (1982) 196.

[10] O. Breitenstein, K. Iwig and I. Konovalov: Physica Status Solidi (a), 160(1), (1997) 271.

[11] O. Breitenstein, M. Langenkamp, O. Lang, A. Schirrmacher: Solar Energy Materials and Solar Cells, 65(1-4), (2001) 55.

[12]D. Shvydka, J. P. Rakotoniaina, O. Breitenstein: Appl. Phys. Lett., 84(5), (2004) 729.

[13] W. Fang, K. Ito: SIAM Journal on Applied Mathematics, 54(4), (1994) 1067.

(Received: June 7, 2011, Accepted: December 20, 2011) 